\title{
Development of Motion Reduction Device for Ship Using Underactuated Parallel Link Mechanism
}

\author{
Akihiro Morinaga, ${ }^{1 *}$ Takahiro Ogawa, ${ }^{1}$ Kakeru Iwanaga, ${ }^{1}$ \\ Yoichi Shimomoto, ${ }^{1}$ and Ikuo Yamamoto ${ }^{2}$ \\ ${ }^{1}$ Graduate School of Engineering, Nagasaki University, \\ Bunkyo-machi 1-14, Nagasaki City, Nagasaki 852-8521, Japan \\ ${ }^{2}$ Organization for Marine Science and Technology, Nagasaki University, \\ Bunkyo-machi 1-14, Nagasaki City, Nagasaki 852-8521, Japan
}

(Received January 6, 2021; accepted February 19, 2021)

Keywords: parallel link mechanism, nonlinear control, offshore device

Transferring between a ship and an offshore platform is potentially dangerous owing to excessive motion caused by rough conditions. In this paper, we focus on a small vessel with consideration of three degrees of freedom of motion: heaving, rolling, and surging. To stabilize these motions using a relatively small device, we propose an underactuated mechanism including two linear actuators and a two-degree-of-freedom gangway connected to the transfer platform. First, we clarify the degrees of freedom and range of the mechanism. Next, we derive a model and design a controller to stabilize the system. Finally, the effectiveness of the mechanism and its controller are tested by numerical simulation as well as by an actual experiment on a small-scale model of the mechanism.

\section{Introduction}

At sea, ships are greatly affected by waves. Therefore, in many ports, breakwaters have been built to reduce the rocking of ships at the docks and to increase safety by preventing waves from coming in from the open sea. However, some remote islands and offshore facilities are not protected by breakwaters, and the waves can cause ships to rock significantly. As an example, Hashima Island, located in Nagasaki and recently placed on the World Cultural Heritage list, is not surrounded by breakwaters. Therefore, the rocking of ships used for tourism is not reduced and directly reflects the sea conditions. Therefore, a rope is used to fix ships to the land. However, such a ship is not well constrained, and, depending on the sea conditions, the rocking of the ship may be uncontrollable, making it impossible to land. Therefore, various studies are being conducted on the analysis and prediction of ship sway. ${ }^{(1,2)}$

Generally, a gangway is commonly used for transfer between ship and land. A gangway is a device attached to the ship side and used as a bridge to the land. It is used in many ships because of its ease of use, but it can only be used in places where the motion of the ship is small. Because of the dangers involved in the use of a gangway in remote islands and offshore

*Corresponding author: e-mail: a-morinaga@nagasaki-u.ac.jp https://doi.org/10.18494/SAM.2021.3258 
power generation facilities, motion reduction devices have been developed for use in places where ships are subjected to significant rocking. Such motion reduction devices are installed on the ship side and reduce the sway of the gangway on which people are moving, allowing a gangway to be used in places where the ship sways greatly. These devices have mainly been commercialized in Europe, where maritime policies are more advanced, and include Momac Offshore Transfer System (MOTS) ${ }^{(3-5)}$ and Ampelmann. ${ }^{(3-5)}$ MOTS is a motion reduction device for serial linkage mechanisms. It is used to access offshore wind power facilities owing to its relatively small size and wide range of motion. However, owing to its low carrying capacity, it is used for transferring a small number of people but is not suitable for transporting cargo. On the other hand, Ampelmann is a parallel link mechanism called a Stewart platform, ${ }^{(6)}$ which is used for various purposes in addition to sea transfer, and it has been used to access offshore wind and oil field facilities. It can carry cargo due to its high rigidity and high carrying capacity, but its range of motion is narrow and its mechanism is large. Both of these large motion reduction devices have advantages and disadvantages, and their mechanisms are selected according to the operating environment, but they can only be installed on large vessels. However, in Japan, relatively small vessels such as fishing boats and tourist boats are often used to access remote islands and maintain offshore power generation facilities, and motion reduction devices for these vessels have not been introduced to the Japanese market. A horizontal sliding system for ship motion stabilizers using a parallel link mechanism has also been studied. ${ }^{(7-9)}$ Although research on motion reduction devices exists, they are not widely used in Japan, and more research is needed for their practical use.

In this paper, a motion reduction device using an underactuated parallel link mechanism that enables space-saving, miniaturization, and cost reduction is focused on. Relatively small vessels such as fishing boats, tourist boats, and offshore wind power maintenance vessels are considered as targets for this device. The number of actuators is reduced by using an underactuated mechanism, which results in reductions in the cost and size of the motion reduction device. The controller of the motion reduction device is designed by using the input linearization technique, and its effectiveness is confirmed in a simulation and experiment using a small-scale device.

\section{Design of Motion Reduction Device}

Two types of mechanism are used in existing motion reduction devices: serial link and parallel link. The parallel link mechanism consists of several links and joints arranged in parallel between the output link and the base. The links are not subjected to a bending moment, and the actuator can be placed close to the base. Also, because it is a closed-loop mechanism, the total output of the actuators becomes the output of the main body, and the errors of the actuators are averaged out, it has higher output and higher accuracy than the serial link mechanism. However, it has structural problems such as a relatively narrow range of motion and interference between the position of the output and the characteristics of the posture, which require the careful selection of design and operation methods. 


\subsection{Underactuated parallel mechanism}

Since this research targets small ships, a small and low-cost motion reduction device that saves space but has sufficient power output to allow people to ride on the ship is required. Also, it is desirable to design the system in such a way that the control error is as small as possible. Therefore, a parallel link mechanism is chosen to achieve high power, rigidity, and accuracy. However, the parallel link mechanism tends to result in a relatively large device, which is a concern for space-saving and miniaturization. Therefore, in this study, we propose an underactuated parallel link mechanism to reduce the number of actuators to save space and reduce the size of the device. Figure 1 shows the proposed inferior-drive parallel link mechanism. The mechanism consists of a rotary joint and a prismatic joint, with the latter used as the actuators. The output plate has three degrees of freedom: heaving, surging, and pitching. The number of degrees of freedom of the mechanism in Fig. 1 is calculated using Grübler's formula: ${ }^{(10)}$

$$
F=d(l-n-1)+\sum_{i=1}^{n} f_{i}
$$

where $F$ is the number of degrees of freedom of the output plate, $d$ is the number of dimensions of the motion space, $l$ is the number of links, $n$ is the total number of joints, and $f_{i}$ is the number of degrees of freedom of the joints. In this mechanism, the number of dimensions of the motion space is $d=3$, the number of links is $l=6$ (one for the base plate, one for the output plate, and two each for the two actuators), the total number of joints is $n=6$ (two for the base plate, two for the output plate, and one each for the two actuators), and the total number of degrees of freedom of the joints is $\sum f_{i}=6$ (one degree of freedom each for the two actuators and one degree of freedom each for the four rotary joints). Substituting these values into Eq. (1), we obtain $F=3$. Thus, the number of actuators in the mechanism can be reduced by introducing an underactuated system. However, the underactuated system has a small area of stability under gravity and easily loses stability due to external disturbances. Therefore, in this study, the underactuated parallel link mechanism is not used as a stand-alone system but as part of a sway reduction system that includes the ship and the land as shown in Fig. 2.

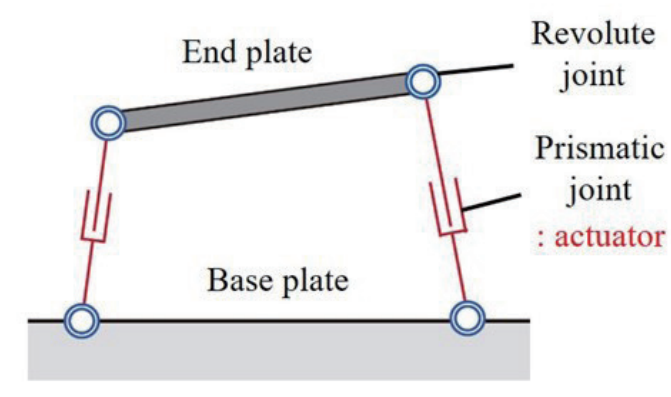

Fig. 1. (Color online) Three-degree-of-freedom parallel mechanism. 


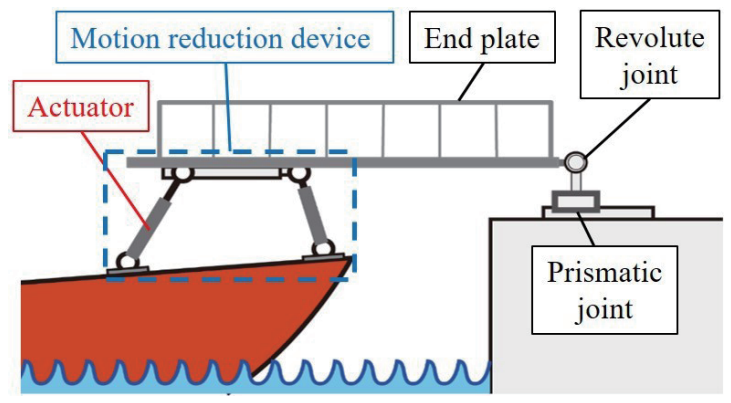

Fig. 2. (Color online) Ship, motion reduction device, gangway, and land.

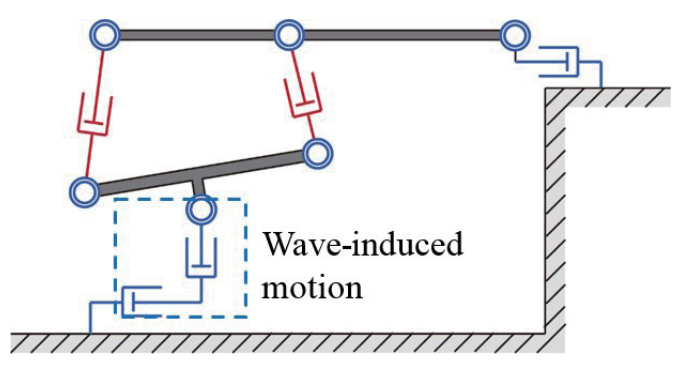

Fig. 3. (Color online) Simplified model.

\subsection{Motion reduction device}

This mechanism uses two linear joints as actuators and a rotational joint to connect the end-plate to the output plate. Also, two linear joints and a rotational joint are used to represent the rocking of the ship, and a linear joint and a rotational joint are used to connect to the land side. In the mechanism shown in Fig. 3, the number of dimensions of the motion space is $d=3$, the number of links is $l=6$, the total number of joints is $n=6$, the total number of degrees of freedom of the joints is six, and the total number of degrees of freedom of the joints is $\sum f_{i}=11$ (one degree of freedom each for the five actuators and one degree of freedom each for the six rotational joints). Substituting these values into Eq. (1), we obtain $F=5$. Thus, this mechanism has five degrees of freedom. For the entire motion reduction system including the ship and the land, the output plate has two degrees of freedom, and the redundancy of the degrees of freedom for the actuator is lost. The position and orientation of the output plate are uniquely determined by the motion of the ship and the length of the actuator. On the other hand, the three degrees of freedom of the ship remain, and the two actuators can cancel the sway of the three degrees of freedom, which means that the design can be realized with fewer parts than a general sway reduction system using a parallel link mechanism.

\section{Design of Controller}

In this section, the design of the control system for the proposed motion reduction system is described. First, the control system of the planar mechanism is designed, and then the position control controller is designed. Finally, the effectiveness of the system is confirmed by simulation.

\subsection{Modeling}

The planar mechanism including the ship and land is represented in Fig. 4. The following eight variables are defined: $l_{L}$ and $l_{R}$ are the lengths of the linear actuators, $\theta$ is the tilt angle from the horizontal plane, $\phi_{L}$ and $\phi_{R}$ are the angles between the linear actuators and base plate, and $\gamma, h$, and $v$ represent the ship's motion. We define a state vector $q$ consisting of the eight variables: 


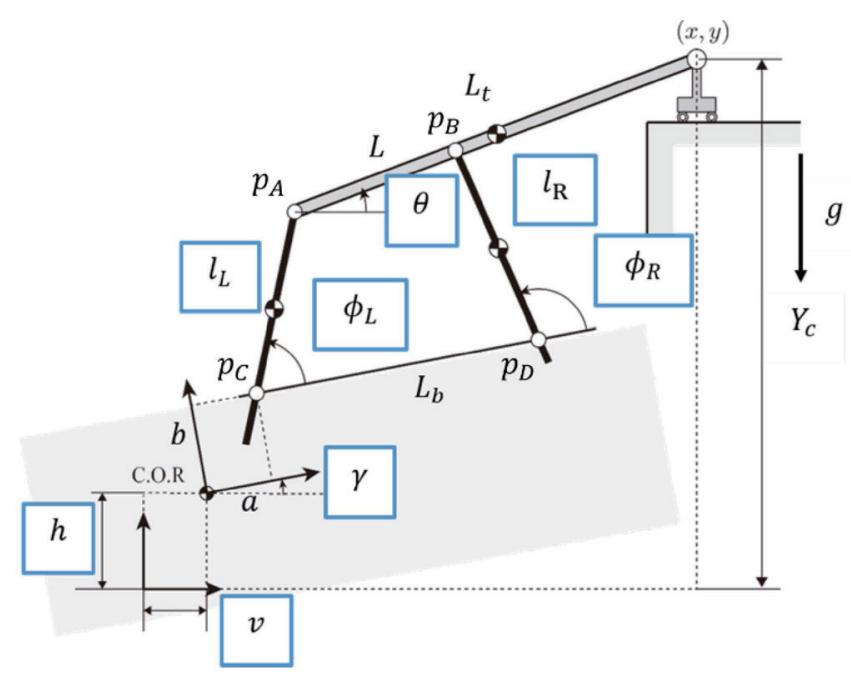

Fig. 4. (Color online) Definition of the coordinate system and variables.

$$
q=\left[l_{L}, l_{R}, \theta, \phi_{L}, \phi_{R}, \gamma, h, v\right]^{T} \text {. }
$$

The total length of the output plate is $L$, the length from the left end of the output plate to the rotation joint is $L_{t}$, the length of the base plate is $L_{b}$, the lengths from the center of gravity of the ship to the actuator connection points are $a$ and $b$, and the vertical distance from the origin of the inertial coordinate system to the output plate is $Y_{c}$. The mechanism has the constraint conditions $\left[\Phi_{1}, \Phi_{2}, \Phi_{3}\right]^{T}=0$, where $\Phi_{1}$ and $\Phi_{2}$ are due to the closed links and $\Phi_{3}$ is due to the constraint on the land side. These constraints can be calculated as

$$
\begin{gathered}
\Phi_{1}=l_{L} \cos \phi_{L}+L_{t} \cos (\theta-\gamma)-l_{R} \cos \phi_{R}-L_{b}=0, \\
\Phi_{2}=l_{L} \sin \phi_{L}+L_{t} \sin (\theta-\gamma)-l_{R} \sin \phi_{R}=0, \\
\Phi_{3}=a \sin \gamma+b \cos \gamma+l_{L} \sin \left(\phi_{L}+\gamma\right)+L \sin \theta+h-Y_{c}=0 .
\end{gathered}
$$

From these equations, the time derivative of the constraint condition is expressed as

$$
\frac{d \Phi(q)}{d t}=A(q) \dot{q}=0
$$

By using Eq. (6) and a matrix $S(q)$ that satisfies $A(q) S(q)=0$, the kinematic model for the combined hull and sway reducer is

$$
\dot{q}=S(q)\left[\begin{array}{l}
u \\
w
\end{array}\right]=F(q) u+G(q) w,
$$


where $u$ is the input and represents the velocity of the actuator, and $w$ is the disturbance and represents the translational velocity and angular velocity of the wave oscillation.

In the position controller design, the actuator length is calculated using the state of the output plate as the target state, and position control is performed using the deviation from the current actuator length. Therefore, the deviation of $e$ from the current position of the actuator to the target length is calculated as

$$
e(t)=\left[\begin{array}{c}
e_{L} \\
e_{R}
\end{array}\right]=\left[\begin{array}{l}
l_{L d}-l_{L} \\
l_{R d}-l_{R}
\end{array}\right],
$$

where $x$ and $\theta$ are the control targets and represent the state of the output plate. Also, $x$ is obtained using the state variables as

$$
x(q)=v+a \cos \gamma-b \sin \gamma+l_{L} \cos (\phi-\gamma)+L \cos \theta .
$$

To linearize Eq. (7), we introduce a virtual input $U$ given by

$$
U=\dot{y}=\frac{\partial h}{\partial q} \dot{q}=H(q) F(q) u+G(q) w
$$

With $U$ as the virtual input, a controller based on proportional-integral-derivative (PID) control is used.

$$
U=K_{p} e(t)+K_{D} \frac{d}{d t} e(t)+K_{I} \int_{0}^{T} e(t) d t
$$

The virtual input is given by Eq. (11). Here, $K_{p}$ is the proportional gain, $K_{D}$ is the derivative gain, and $K_{I}$ is the integral gain. In practice, assuming that the speed can be controlled inside the linear actuator, the following equation is used for control using the input $u$ :

$$
u=\{H(q) F(q)\}^{-1}\{U-\{H(q) G(q) w\}\} .
$$

\subsection{Simulation}

To verify the effectiveness of the control system design, a simulation is performed using MATLAB. In the simulation, the ship is subjected to sway, and the goal of the control is to maintain the position and attitude of the output plate in the initial state. We perform the simulation using the same parameters as those used in the experiment in Sect. 4. The initial position of the output plate is set to $x_{0}=1350 \mathrm{~mm}$ and the initial posture is set to $\theta=0 \mathrm{rad}$. The initial lengths of the actuators are set to $400 \mathrm{~mm}$, which is at the middle of the stroke. The ship's 
oscillations are given by $h(t)=30 \cos (0.2 \pi t+0.5 \pi)$ and $\gamma(t)=v(t)=0$. The simulation results are shown in Figs. 5-8. The figures show the time histories of lengths of the actuators (Fig. 5), the surging of the end-plate (Fig. 6), the heaving of the end-plate (Fig. 7), and the pitching of the end-plate (Fig. 8). The figures show that the state of the output plate remains near the target value by extending and retracting the two actuators in the same direction for vertical sway (heaving), thus confirming the effectiveness of the controller.

\section{Experiment and Discussion}

\subsection{Design of a small-scale experimental device}

An inverse kinematics calculation is used to analyze the range of motion that the device can handle and to determine the mechanical dimensions $L_{t}$ and $L_{b}$. To calculate the length of the actuators, we need to perform inverse kinematics calculations using the state of the output plate and the sway of the ship. First, positions $p_{A}, p_{B}, p_{C}$, and $p_{D}$ in Fig. 4 are calculated as follows.

$$
\begin{gathered}
p_{A}=\left[X_{0}-L \cos \theta, Y_{c}-L \sin \theta\right] \\
p_{B}=\left[X_{0}-\left(L-L_{t}\right) \cos \theta, Y_{c}-\left(L-L_{t}\right) \sin \theta\right] \\
p_{C}=[v-\alpha \cos \gamma-\beta \sin \gamma, h-\alpha \sin \gamma+\beta \cos \gamma] \\
p_{D}=\left[v-\left(\alpha-L_{b}\right) \cos \gamma-\beta \sin \gamma, h-\left(\alpha-L_{b}\right) \sin \gamma+\beta \cos \gamma\right]
\end{gathered}
$$

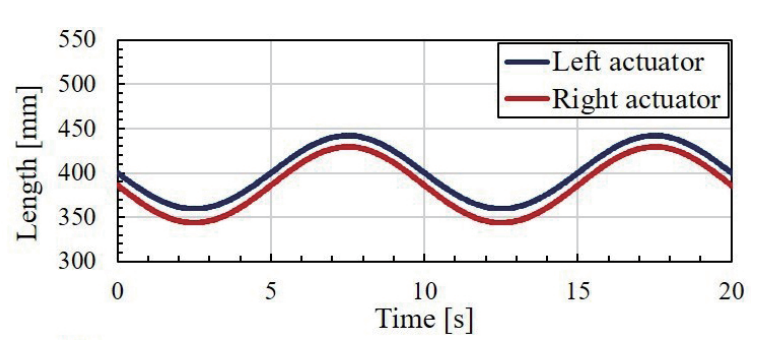

Fig. 5. (Color online) Time histories of lengths of left (blue) and right (red) actuators.

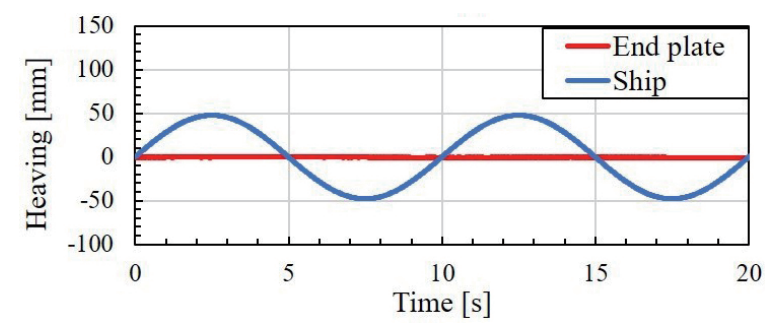

Fig. 7. (Color online) Time histories of heaving of end-plate (red) and ship (blue).

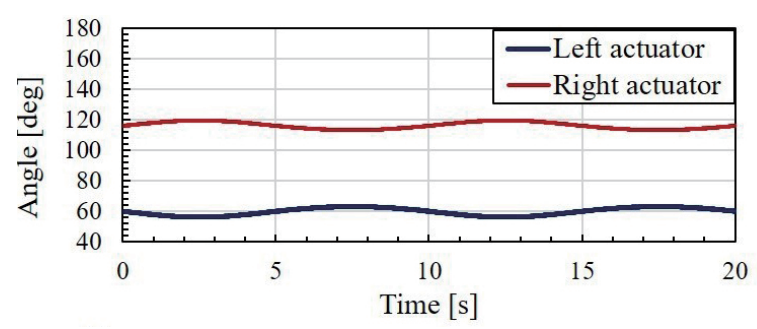

Fig. 6. (Color online) Time histories of surging of end-plate (red) and ship (blue).

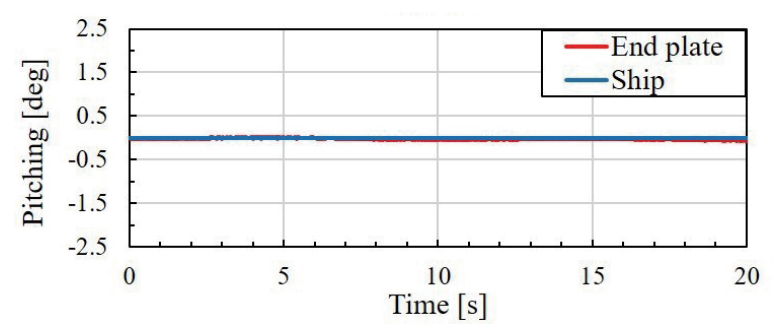

Fig. 8. (Color online) Time histories of pitching of end-plate (red) and ship (blue). 
From the above equations, the length of the actuators can be calculated as $l_{L}=\left\|P_{A}-P_{C}\right\|$ and $l_{R}=\left\|P_{B}-P_{D}\right\|$. Next, the stroke limits of the actuators $l_{L \min } \leq l_{L d} \leq l_{L \max }$ and $l_{R \min } \leq l_{R d} \leq l_{R \max }$ and the angular limits $\phi_{L \min } \leq \phi_{L d} \leq \phi_{L \max }$ and $\phi_{R \min } \leq \phi_{R d} \leq \phi_{R \max }$ are given. The dimensions of the link parameters are determined to maximize the ranges of $\gamma, h$, and $v$ that satisfy the above limitations. The range of realized motion of the ship is visualized by plotting only when the stroke and angular limits are satisfied by sequentially shaking the ship.

From the conditions of the experimental environment, the length of the top plate is initially set to $L=1500 \mathrm{~mm}$, and from the dimensions of the actuator used, the limitations of the lengths of the actuators are $l_{L \min }=L_{R \min }=330 \mathrm{~mm}$ and $l_{L \max }=L_{R \max }=470 \mathrm{~mm}$, and the limitations of the angles are $\phi_{\text {Lmin }}=\phi_{R \min }=30 \mathrm{deg}$ and $\phi_{\text {Lmax }}=\phi_{R \max } 150 \mathrm{deg}$. We simulate the combinations of $L_{t}=300,500$, and $700 \mathrm{~mm}$ and $L_{b}=300,500$, and $700 \mathrm{~mm}$. The results of some simulations are shown in Figs. $9\left(L_{t}=300 \mathrm{~mm}\right.$ and $\left.L_{b}=300 \mathrm{~mm}\right)$ and $10\left(L_{t}=300 \mathrm{~mm}\right.$ and $\left.L_{b}=700 \mathrm{~mm}\right)$. From the results, it can be seen that as the length of the upper part of the link $L_{t}$ decreases and the length of the lower part of the link $L_{b}$ increases, the range of heaving increases. Therefore, in this study, the dimensions of the link mechanism are set to $L_{t}=300 \mathrm{~mm}$ and $L_{b}=700 \mathrm{~mm}$.

\subsection{Experiment}

We conduct a simple experiment in which only the vertical motion of the ship is given. After the start of the experiment, the state is obtained by sensor measurement and calculation from
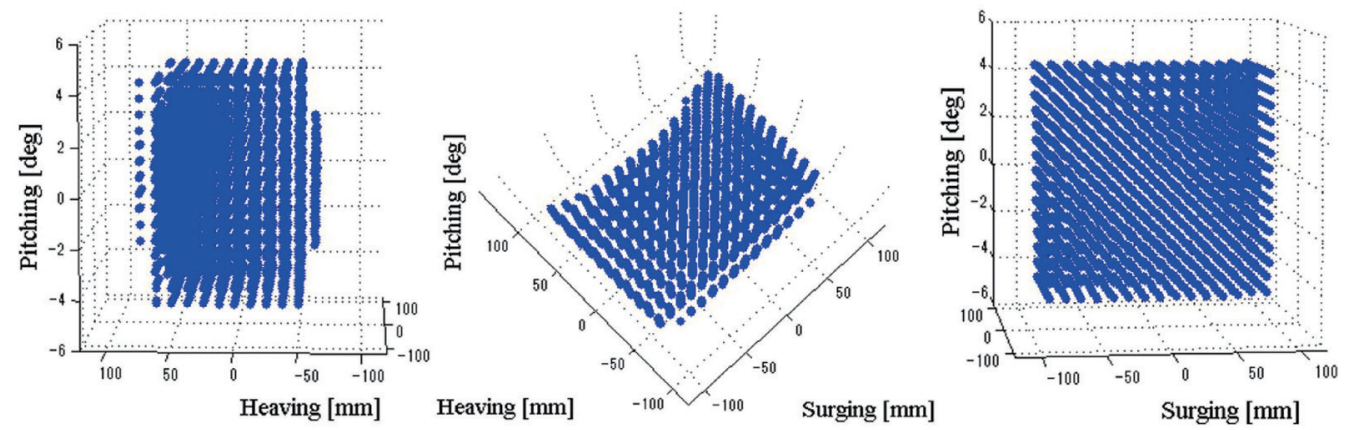

Fig. 9. (Color online) Result for $\boldsymbol{L}_{\boldsymbol{t}}=300 \mathrm{~mm}$ and $\boldsymbol{L}_{\boldsymbol{b}}=300 \mathrm{~mm}$.
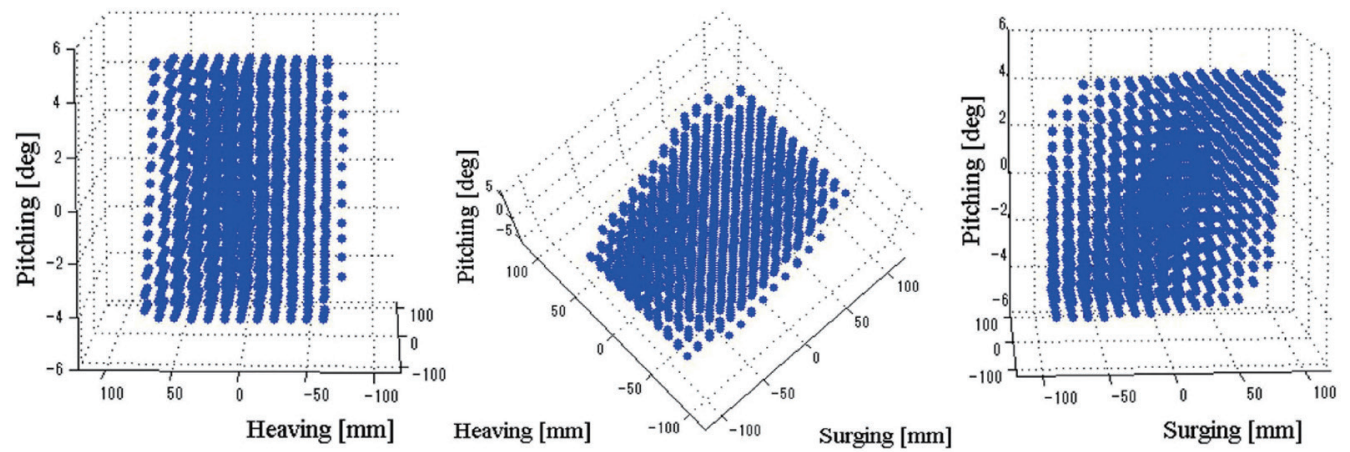

Fig. 10. (Color online) Result for $\boldsymbol{L}_{\boldsymbol{t}}=300 \mathrm{~mm}$ and $\boldsymbol{L}_{\boldsymbol{b}}=700 \mathrm{~mm}$. 
the constraint conditions, and the target position of the actuator is calculated to determine the input. The angles of the top and base plates, $\theta$ and $\gamma$, respectively, and the longitudinal sway of the ship $v$ are measured by a tilt sensor. The lengths of the actuators, $l_{L}$ and $l_{R}$, are measured by potentiometers mounted on the actuators. The position of the output plate is measured using a linear motion measuring device. From these measurements, the forward/backward motion $v$ and vertical motion $h$ of the ship are calculated using Eqs. (5) and (9). The experimental setup is shown in Fig. 11.

The experimental results are shown in Figs. 12-15. The figures show the time histories of the lengths of the actuators (Fig. 12), the surging of the end-plate (Fig. 13), the heaving of the end-plate (Fig. 14), and the pitching of the end-plate (Fig. 15). According to Fig. 14, the change in the vertical direction of the output plate is reduced to $30 \mathrm{~mm}$ while the vertical sway of the ship (heaving) is $100 \mathrm{~mm}$. This satisfies the target of reducing the height of the output plate to $50 \mathrm{~mm}$ or less.

In this experiment, we were able to reduce the sway of the output plate by half compared with the vertical sway given to the ship. This indicates that the sway reduction system has the

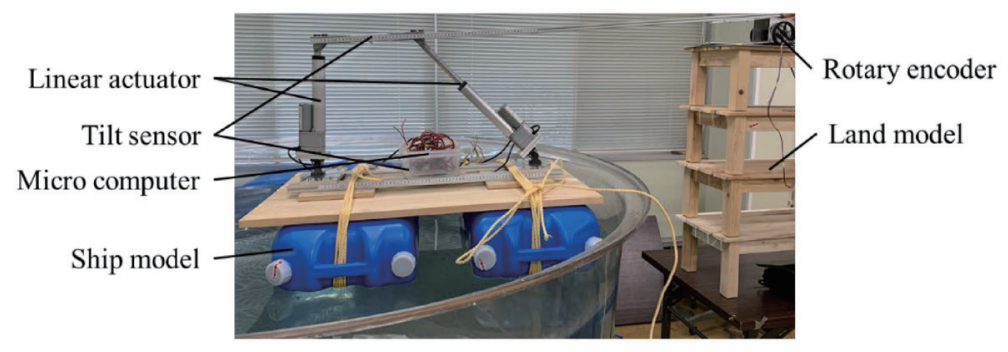

Fig. 11. (Color online) Experimental setup.

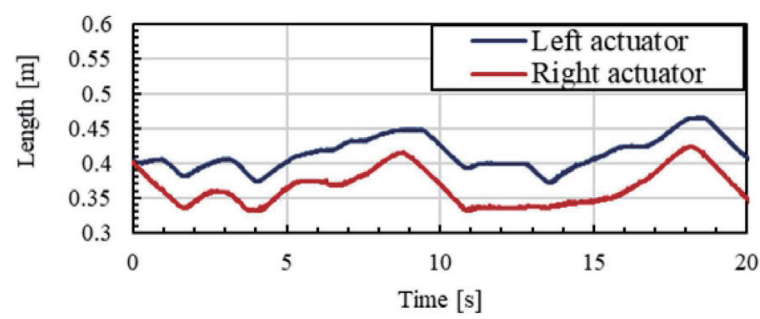

Fig. 12. (Color online) Time histories of lengths of left (blue) and right (red) actuators.

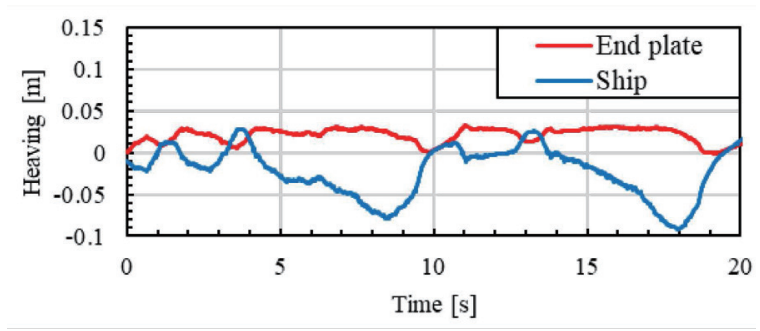

Fig. 14. (Color online) Time histories of heaving of end-plate (red) and ship (blue).

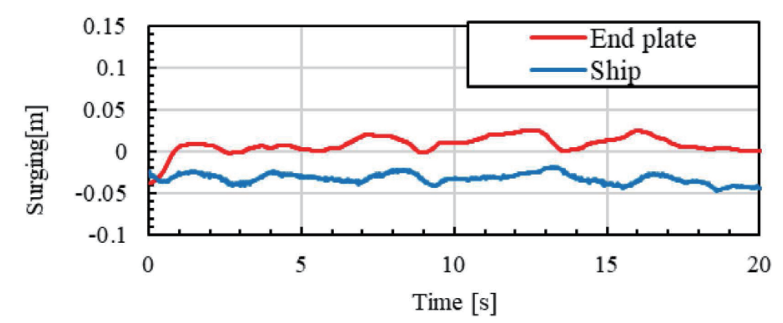

Fig. 13. (Color online) Time histories of surging of end-plate (red) and ship (blue).

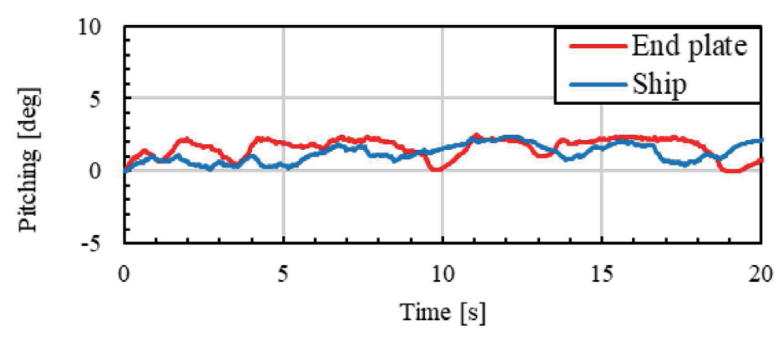

Fig. 15. (Color online) Time histories of picthing of end-plate (red) and ship (blue). 
targeted reduction performance. However, it was not able to maintain the target attitude as in the simulation. There are several reasons for this: the sensor used in the device may not have acquired the correct state due to large errors; the speed of the sway given in the experiment may have been beyond the performance of the actuator; and the actuators may have extended or contracted to the stroke limit, resulting in a loss of controllability. Therefore, it is necessary to reduce the error of the data obtained from the sensor and to review the actuators. Also, it is necessary to design a ship that is easily shaken to make the shaking of the ship in the experiment closer to that in the simulation.

\section{Conclusions}

In this paper, a marine transfer system using an inferior-drive parallel link mechanism was proposed for use on small vessels as an access method to power generation facilities in underserved ports and offshore facilities. As a basic study for this system, the control design of a sway reduction device with three degrees of freedom in the plane was conducted. Finally, the effectiveness of the proposed system was confirmed in the experiment by the fact that the oscillation of the output plate was suppressed to the target value when heaving was given to the ship model. In the future, it is necessary to improve the design to accommodate more degrees of freedom and to be able to handle larger shaking by increasing the range of motion of the motion reduction device. In addition, the effects of mooring ropes and anchors on the ship during berthing should be considered, and more accurate modeling and a corresponding controller should be designed.

\section{Acknowledgments}

This work was supported by JSPS KAKENHI Grant Number 17K14618.

\section{References}

1 C. Gilbert, J. Browell, and D. McMillan: J. Phys. 1222, WindEurope Conference and Exhibition 2019 (2-4 April 2019). https://iopscience.iop.org/article/10.1088/1742-6596/1222/1/012040/pdf

2 M. Hamaguchi, N. Kimura, T. Hokimoto, J. Kawasaki, and K. Amagai: Proc. Japan Institute of Navigation Conf. 98 (1998) 396. https://doi.org/10.9749/jin.98.369

3 L. B. Savenije, G. Katsouris, and E. C. Nederland: Offshore Wind Access (TNO Publications, 2017). http:// resolver.tudelft.nl/uuid:547fcb60-2a93-489b-9db0-7fbf2aef404b

4 H. P. Stumpf and B. Hu: Offshore Wind Access (ECN, 2018). https://publicaties.ecn.nl/PdfFetch. aspx?nr=ECN-E--17-071

5 B. Hu, P. Stumpf, and W. van der Deijl: Offshore Wind Access (TNO, 2019). http://resolver.tudelft.nl/ uuid:e8f05155-aa5a-4aad-a7ba-8bed2e9b08fe

6 D. Stewart: Proc. Institution of Mechanical Engineers 180 (1965) 371. https://doi.org/10.1243/PIME PROC 1965 180_029 02

7 Y. Zhang, E. Shimizu and M. Ito: Proc. Japan Society of Mechanical Engineers (2008). https://doi.org/10.1299/ jsmekanto.2008.14.179

8 Y. Zhang, E. Shimizu and M. Ito: J. Marine Eng. 44 (2009) 316. https://doi.org/10.5988/jime.44.316

9 Y. Zhang, F. Zhang, and M. Ito: Marine Eng. 45 (2010) 725. https://doi.org/10.5988/jime.45.725

10 J. J Craig: Introduction to Robotics: Mechanics and Control (Prentice-Hall, 2009). 